\title{
Déclaration sur la protection contre l'encéphalite japonaise
}

\section{Préambule}

Le Comité consultatif de la médecine tropicale et de la médecine des voyages (CCMTMV) fournit à l'Agence de la santé publique du Canada (ASPC), de façon continue et en temps utile, des conseils de nature médicale, scientifique et de santé publique concernant les maladies tropicales infectieuses et les risques pour la santé associés aux voyages à l'étranger. L'ASPC reconnaît que les conseils et les recommandations figurant dans cette déclaration reposent sur la pratique médicale et les connaissances scientifiques les plus à jour, et les diffuse dans le but d’informer les voyageurs ainsi que les professionnels de la santé qui sont appelés à leur prodiguer des soins.

Les personnes qui administrent ou utilisent des médicaments, des vaccins ou d'autres produits devraient bien connaître le contenu des monographies de produit ou des autres normes ou modes d'emploi approuvés. Les recommandations relatives à l'usage des produits et les autres renseignements présentés ici peuvent différer de ceux qui figurent dans la monographie ou toute autre norme ou instruction approuvée et pertinente établie par les fabricants autorisés. Rappelons que l'approbation demandée par les fabricants pour leurs produits, avec démonstration de leur innocuité et de leur efficacité à l'appui, ne s'applique qu'aux utilisations conformes à la monographie ou autre norme ou mode d'emploi approuvé.

\section{Introduction}

L'encéphalite japonaise (EJ) est causée par un flavivirus transmis par des moustiques du genre Culex. Ce virus est l'une des principales causes d'encéphalite virale dans le monde : selon les estimations, on répertorie chaque année au moins 50000 cas d'EJ, 10000 décès par cette maladie et 15000 cas qui subissent des séquelles neuropsychiatriques permanentes ${ }^{(1)}$. L'EJ frappe de nombreuses régions d'Asie, en particulier les régions du Sud-Est et certaines parties du Pacifique Ouest (voir les annexes $\mathrm{A}$ et $\mathrm{B})^{(1)}$. Dans les zones rurales où il y a transmission active de l'EJ, la proportion de cas possibles dans la population résidente réceptive est de 0,1 à 2 cas pour 100000 habitants par semaine ${ }^{(2)}$. Il n'existe pas de traitement spécifique contre l'EJ ${ }^{(3)}$, mais l'immunisation au moyen d'un vaccin efficace ${ }^{(4,5)}$ et le recours à des mesures de protection individuelle (MPI) ${ }^{(6)}$ offrent une protection substantielle contre l'infection et la maladie.

\footnotetext{
*Membres : Dr P.J. Plourde (président); Dr C. Beallor; Dr A. Boggild; Dr J. Brophy; Dr M. Crockett; Dr W. Ghesquiere; Ms A. Henteleff; Dr A. McCarthy; Dr K. L. McClean Représentants d'office : Dr G. Brunette, Dr J. Creaghan, Dr P. Charlebois; Dr M. Tepper; Dr P. McDonald; Dr J. Given Représentants de liaison : Dr C. Greenaway; Dr A. Pozgay; Dr C. Hui; Dr P. Teitelbaum

Membre émerite : DrC. William L. Jeanes

Consultant : Dr S. Schofield

† La présente déclaration a été préparée par Dr M. Tepper et Dr S. Schofield et approuvée par le CCMTMV
} 


\section{Tableau clinique}

La maladie symptomatique ne se développe que chez une faible proportion des humains infectés par le virus de l'EJ (proportion signalée : 1/25 à 1/1 000)(7), après une période d'incubation de 5 à 15 jours. Le taux de létalité parmi les cas d'infection symptomatique à virus de l'EJ (VEJ) est d'environ 20 à $30 \%$, et, parmi les survivants, 30 à $50 \%$ sont aux prises avec des séquelles neurologiques ou psychologiques permanentes $^{(3)}$. Dans les régions endémiques, la maladie touche surtout les enfants ${ }^{(7)}$; la plupart des adultes présentent des preuves sérologiques d'infection antérieure à VEJ.

\section{Epidémiologie}

C'est dans les zones rurales agricoles que l'EJ constitue surtout une menace. Le virus se perpétue dans un cycle enzootique dans lequel interviennent des moustiques du genre Culex et des oiseaux sauvages (p. ex. des ardéidés tels les aigrettes et les hérons) $)^{(4)}$. Le cycle épizootique secondaire peut mener à l'infection d'hôtes accidentels, comme l'humain et le cheval, et le porc joue souvent le rôle d'hôte amplificateur.

Les moustiques du genre Culex constituent le principal vecteur de l'EJ. L'espèce surtout en cause est Culex tritaeniorhynchus, qui pique principalement à l'aube et à la brunante, plus souvent à l'extérieur qu'à l'intérieur, et qui préfère les mammifères autres que l'humain. Les larves de cette espèce et de plusieurs autres espèces du genre Culex se développent dans les eaux stagnantes, et les rizières peuvent être un habitat important parce qu'elles peuvent héberger de très grandes populations de larves ${ }^{(4)}$.

Il existe deux profils épidémiologiques d'EJ. Dans les régions tempérées, on observe une transmission sporadique et des épidémies saisonnières périodiques (été et automne), alors que, dans les régions subtropicales ou tropicales, la transmission peut survenir essentiellement tout au long de l'année ${ }^{(1,4)}$. Prière de se reporter à l'annexe A pour voir la description du risque d'EJ par pays et à l'annexe B pour voir une carte des régions à risque d'EJ.

Les programmes de vaccination à grande échelle contre l'EJ (ciblant les enfants), les modifications des pratiques d'élevage et l'urbanisation accrue ont entraîné une réduction majeure du nombre de cas humains d'EJ dans certains pays ${ }^{(1,4)}$, par exemple le Japon et la Corée. Cependant, l'EJ peut toujours poser un risque pour les personnes non immunisées (p. ex. voyageurs) dans ces pays parce que la transmission peut s'y poursuivre dans un cycle zoonotique.

On a répertorié peu de cas d'EJ parmi les voyageurs occidentaux. Dans les documents publiés, seuls 55 cas d'EJ associée aux voyages ont été signalés chez les personnes non originaires d'un pays d'endémie dans la période de 35 ans s'échelonnant entre 1973 et 2008; ces cas étaient constitués de 33 touristes, neuf expatriés, six soldats et sept personnes dont le motif du voyage est inconnu ${ }^{(8)}$. Dans les données publiées par le réseau de surveillance GeoSentinel, qui portaient sur près de 50000 patients et qui ont été recueillies dans plus de 40 cliniques spécialisées en médecine des voyages et en médecine tropicale réparties sur six continents, aucun cas d'EJ n'a été déclaré(9; 10); cependant, seule une partie des voyageurs du réseau se sont rendus dans une région à risque d'EJ. En 1982, un cas possible d'EJ a été signalé, soit un Canadien de retour de la Mandchourie (nord-est de la Chine) ${ }^{(11)}$; il pourrait s'agir du seul cas canadien jamais signalé. Chez certains cas associés aux voyages, le séjour en milieu rural avait été court, par exemple une seule nuit ${ }^{(12)}$. Le risque global estimé d'EJ clinique parmi les voyageurs ayant séjourné pendant une courte période en Asie est inférieur à 1 pour 1 million $^{(3)}$, mais, dans les zones de forte endémie pendant la saison de transmission, le risque peut atteindre celui observé dans la population résidente réceptive $(0,1$ à 2 pour 100000 par semaine) $)^{(2)}$.

L'estimation précise du risque pour un voyageur non protégé d'être exposé à l'EJ ou de contracter la maladie est très difficile et est tributaire de paramètres tels que la destination, la durée et la saison du voyage et les activités pratiquées; prière de se reporter à l'annexe $\mathrm{C}$ pour obtenir des conseils relativement à l'évaluation du risque d'EJ chez un voyageur.

\section{Mesures de protection individuelle}

Les insectifuges contenant du DEET ( $N, N$-diéthyl-m-toluamide $)^{(13,14)}$, les moustiquaires de lit traitées à la perméthrine ${ }^{(6,15)}$ et les vêtement traités à la perméthrine ${ }^{(16)}$ se sont révélés efficaces pour prévenir les piqûres des moustiques du genre 
Culex. En effet, l'utilisation de moustiquaires de lit traitées a permis à elle seule une réduction considérable des cas d'EJ ${ }^{(6)}$. Globalement, les MPI bien appliquées devraient réduire de façon marquée le risque d'exposition à l'EJ ${ }^{(17)}$ (et à d'autres maladies transmises par des arthropodes comme la dengue); la réduction pourrait être de l'ordre de $90 \%$.

\section{Vaccin contre l'encéphalite japonaise}

Jusqu'à tout récemment, le seul vaccin contre l'EJ offert au Canada était le vaccin inactivé JE-VAX ${ }^{\mathrm{MD}}$, produit par Biken (Fondation pour la recherche sur les maladies microbiennes de l'Université d'Osaka) au moyen de cerveaux de souris et distribué par Sanofi Pasteur Limited. Toutefois, ce vaccin n'est plus produit ni commercialisé et n'est donc plus disponible au Canada.

La vente d'un vaccin inactivé produit par culture du virus sur cellules Vero, IXIAROMD ${ }^{\mathrm{M}}$, qui est fabriqué par Intercell AG et commercialisé au Canada par Novartis Pharmaceuticals Canada Inc, a été autorisée au Canada en décembre 2009(18); il s'agit du seul vaccin contre l'EJ actuellement disponible au Canada. Il est important pour ceux qui prescrivent ou inoculent IXIAROMD de lire la monographie de produit ${ }^{(19)}$. Parmi les caractéristiques importantes d'IXIARO ${ }^{\mathrm{MD}}$, mentionnons les suivantes :

- Administration : IXIAROMD est administré par voie intramusculaire. Le schéma de primovaccination comporte deux doses de $6 \mu \mathrm{g}$ chacune administrées à 0 et 28 jours. Il n'existe pas de schéma « rapide ou accéléré ».

- Âge à partir duquel l'utilisation du vaccin est autorisée : Lutilisation d'IXIAROMD n'est autorisée que pour les personnes de 18 ans ou plus ${ }^{(18,19)}$. Cette autorisation assortie d'une limite d'âge et la non-disponibilité de JE-VAX ${ }^{\mathrm{MD1}}$ créent un problème majeur si l'on souhaite offrir une protection vaccinale aux personnes de moins de 18 ans. Il n'existe actuellement aucun moyen satisfaisant de protéger les personnes de moins de 18 ans contre l'EJ. Chez le très petit nombre d'enfants

1 Selon des discussions tenues avec Sanofi Pasteur au Canada et aux États-Unis, l'importation de JE-VAX'Mo des États-Unis n'est pas une option, car les stocks des É.-U. sont destinés expressément aux personnes de moins de 17 ans et seront périmés au printemps 2011.
(40 à 50) qui ont reçu deux doses d'IXIARO ${ }^{\mathrm{MD}}$, le vaccin a provoqué la formation d'anticorps protecteurs comme chez les adultes sans provoquer d'événements indésirables inattendus ${ }^{(3,19,20)}$. Ladministration d'une demi-dose ( $3 \mu \mathrm{g}$ au lieu de $6 \mu \mathrm{g}$ ) a aussi été envisagée pour les jeunes enfants ${ }^{(20)}$. La Food and Drug Administration (FDA) des États-Unis a autorisé l'utilisation d'IXIARO $^{\mathrm{MD}}$ chez les 17 ans et plus ${ }^{(21)}$, alors que l'Agence européenne des médicaments (EMA) ${ }^{(22)}$, tout comme Santé Canada ${ }^{(18)}$, a autorisé son utilisation chez les 18 ans et plus. Si un voyage ne peut être évité ni reporté, il faudrait recommander aux voyageurs de moins de 18 ans d'utiliser de façon assidue les MPI. En ce qui concerne la vaccination, il est reconnu que plusieurs approches peuvent être envisagées, par exemple l'utilisation d'IXIARO ${ }^{\mathrm{MD}}$ de façon « non conforme à l'étiquette » ou l'immunisation au moyen d'un vaccin offert dans la région à risque, auquel cas il faut soupeser avec soin les risques et les avantages.

- Efficacité : Lutilisation d'IXIAROMD est autorisée en fonction d'une réponse sérologique non inférieure à celle de JE-VAX ${ }^{\mathrm{MD}(19,23)}$ et d'un seuil, établi par l'Organisation mondiale de la santé, de $\geq 1$ :10 des anticorps neutralisants ${ }^{(1)}$, et non pas en fonction d'une prévention bien établie de la maladie chez l'humain. Toutefois, les organismes d'homologation du Canada (Santé Canada ${ }^{(18)}$ ) et d'ailleurs (p. ex. FDA des É.-U. ${ }^{(21)}$, EMA de l'Union européenne $\left.{ }^{(22)}\right)$ estiment que les données sont suffisamment robustes pour associer une réponse sérologique démontrée et la prévention de la maladie.

- Événements indésirables : À ce jour, d'après le nombre relativement faible de vaccinés dans le cadre d'essais cliniques ou dans d'autres circonstances, IXIARO ${ }^{\mathrm{MD}}$ n'est pas associé à un profil d'événements indésirables inquiétants comparativement à JE-VAX ${ }^{\mathrm{MD}}$ ou à un placebo $^{(3,19,24)}$. Toutefois, seule la surveillance postcommercialisation permettra d'évaluer le profil complet des événements indésirables, en particulier ceux qui sont rares.

- Grossesse et allaitement : Il n'existe pas de données concernant l'innocuité (ni l'efficacité) d'IXIARO ${ }^{\mathrm{MD}}$ chez les femmes enceintes et celles qui allaitent ${ }^{(3,19)}$, et il n'est donc recommandé d'utiliser ce vaccin chez de telles femmes que lorsque le risque de maladie l'emporte sur 
le risque (inconnu) pour la femme, son foetus ou l'enfant

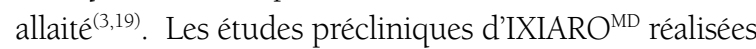
chez des rates gravides n'ont pas révélé de risque pour la mère ou son foetus attribuable au vaccin ${ }^{(19)}$.

- Production d'anticorps: Par suite de l'injection d'une dose unique d'IXIARO ${ }^{\mathrm{MD}}$, on observe la présence d'un titre suffisant d'anticorps protecteurs chez $30 \%$ des vaccinés 10 jours après la vaccination et chez $40 \%$ des vaccinés 28 jours après la vaccination ${ }^{(3,19)}$. Lorsqu'une deuxième dose est administrée 28 jours après la première dose, on observe 28 jours plus tard la présence d'anticorps chez environ $95 \%$ des vaccinés ${ }^{(3,19,23)}$. Il faut généralement sept jours après la vaccination pour que les anticorps fassent leur apparition ${ }^{(19)}$; par conséquent, il est préférable que le délai entre la vaccination et l'arrivée dans la zone à risque d'EJ soit suffisamment long. Si le délai est trop court pour qu'il soit possible d'administrer les deux doses de la série vaccinale, une dose unique peut être envisagée étant donné qu'une bonne minorité des vaccinés peuvent produire des anticorps protecteurs ou que cette dose peut stimuler le système immunitaire du vacciné de façon qu'il réponde à une exposition naturelle à l'EJ en temps opportun. Ladministration simultanée de deux doses du vaccin pourrait porter le taux de séroconversion à $60 \% 10$ jours après la vaccination ${ }^{(25)}$; cependant, il s'agirait alors d'une administration «non conforme à l'étiquette ».

- Dose de rappel : Lors des essais cliniques menés à ce jour, le titre des anticorps protecteurs produits par suite de l'administration d'IXIARO ${ }^{\mathrm{MD}}$ déclinait avec le temps. Une proportion de 80 à $95 \%$ des vaccinés ayant reçu la série vaccinale complète avaient toujours un titre suffisant 6 mois après la première dose, et 60 à $80 \%$ avaient toujours un titre suffisant 12 mois après la première dose $e^{(3,19,26,27)}$. Une « dose de rappel »du vaccin induit la production d'anticorps protecteurs à un titre suffisant chez ceux dont les anticorps protecteurs ont disparu 12 mois après la première dose $e^{(3,19,26)}$. Pour le moment, il est impossible de recommander un intervalle précis avant l'administration d'une dose de

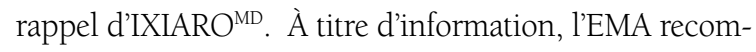
mande l'administration d'une dose de rappel 12 à 24 mois après la série vaccinale primaire si le sujet risque d'être exposé de nouveau à l'EJ ou après 12 mois si le risque est permanent (p. ex. résidence dans une région endémique) ${ }^{(22)}$; ces intervalles semblent raisonnables.
- Interchangeabilité : Il n'existe pas de données en ce qui concerne l'interchangeabilité d'IXIARO ${ }^{\mathrm{MD}}$ et de $\mathrm{JE} \mathrm{VAX}^{\mathrm{MD}}$, que ce soit pour la primovaccination ou l'administration de la dose de rappel ${ }^{(3)}$. En l'absence de données, il est recommandé de procéder comme suit : si un patient a reçu JE-VAX ${ }^{\mathrm{MD}}$ il y a plus de 3 ans et qu'il doit encore (ou de nouveau) être protégé contre l'EJ, il devrait recevoir deux doses d'IXIARO ${ }^{\text {MD }}$ (c.-à-d. la série vaccinale primaire). IXIARO ${ }^{\mathrm{MD}}$ et JE-VAX ${ }^{\mathrm{MD}}$ ciblent différentes souches du VEJ, c'està-dire $\mathrm{SA}_{14}$-14-2 (IXIAROMD) et Nakayama-NIH $\left(J E-V A X^{M D}\right)$; cette différence en ce qui concerne les souches ciblées pourraient expliquer certaines des différences relevées lors des comparaisons des titres d'anticorps mesurés avec les deux vaccins; on ignore si ces différences présentent un intérêt clinique.

- Administration concomitante : Les effets de l'administration concomitante d'IXIAROMD et d'un autre vaccin n'ont été étudiés qu'avec un seul autre vaccin (HAVRIX ${ }^{\mathrm{MD}}$ ), et la réponse immunitaire (formation d'anticorps) à chaque vaccin a correspondu à celle attendue $e^{(3,19,28)}$. Dans le groupe ayant reçu les deux vaccins en concomitance, on a signalé d'avantage de douleur, de rougeur et d'enflure que dans les groupes ayant reçu un seul vaccin. En règle générale, l'administration de plusieurs vaccins lors de la même séance n'est pas contre-indiquée si ces vaccins sont administrés « séparément », par exemple au moyen de seringues différentes ou à un point d'injection ou dans un membre différent ${ }^{(29)}$.

- Autres études : Les essais cliniques futurs devraient apporter des réponses à bon nombre des questions abordées dans les paragraphes précédents, par exemple l'utilisation du vaccin chez les enfants, la possibilité d'un calendrier accéléré et les doses de rappel.

- Décision de recommander ou non le vaccin : La décision de recommander ou non le vaccin contre l'EJ devrait reposer sur le degré très variable de transmission, les mesures préventives de remplacement (comme les MPI), la tolérance individuelle au risque et le rapport cout-efficacité. Vu le coût élevé d'IXIARO ${ }^{\mathrm{MD}}$, son administration pourrait ne pas être jugée particulièrement rentable lorsque la transmission est faible ou très faible ou lorsque d'autres stratégies de prévention, dont les MPI, sont suivies à la lettre ${ }^{(3)}$. 


\section{Recommandations du CCMTMV}

\section{Recommandations du CCMTMV}

1. Les $\mathrm{MPI}^{(6,13,14,15,16,17)}$ bien utilisées réduisent le risque d'EJ et sont recommandées. Lorsque le risque d'EJ est faible, le recours aux MPI devrait abaisser le risque déjà faible à un niveau auquel IXIARO ${ }^{\mathrm{MD}}$ n'offre que peu de bienfaits additionnels. Par exemple, en présence d'un risque d'infection à VEJ de 1/10 000 sans aucune protection, des MPI efficaces à $90 \%$ portent à 1/100 000 le risque résiduel qu'IXIARO ${ }^{\mathrm{MD}}$ pourrait réduire.

2. IXIARO ${ }^{\mathrm{MD}}$ devrait prévenir efficacement l'EJ.

3. En règle générale, IXIARO ${ }^{\mathrm{MD}}$ est recommandé pour les séjours dans une région où l'EJ est endémique ou épidémique, pendant la saison à risque

i. pour tous les voyageurs qui passeront au total plus de 30 jours dans une zone rurale (ou dans une zone urbaine où l'on sait que l'EJ sévit de façon endémique ou épidémique); parmi ces voyageurs, on compte ceux qui voyagent pendant une longue période et les expatriés qui, bien qu'ils résident principalement en zone urbaine, peuvent faire de courts séjours par intermittence dans des zones rurales à risque;

ii. pour les voyageurs qui passeront au total moins de 30 jours en zone rurale (ou dans une zone urbaine où l'on sait que l'EJ sévit de façon endémique ou épidémique), s'ils prévoient pratiquer globalement beaucoup d'activités extérieures (ou intérieures si le milieu intérieur n'est pas exempt de moustiques), en particulier le soir ou la nuit.

\section{Catégorie MFP(30)}

BI

AI

CIII

CIII

CIII

CIII

CIII

5. Si l'on ne dispose pas d'assez de temps avant l'arrivée dans une zone à risque pour administrer le vaccin conformément au calendrier habituel (à 0 et 28 jours), l'administration d'une dose unique d'IXIARO ${ }^{\mathrm{MD}}$ peut être envisagée si le patient comprend bien que la protection contre l'EJ ne peut pas être garantie. On peut aussi envisager d'administrer simultanément deux doses d'IXIAROMD (utilisation «non conforme à l'étiquette »); dans un tel cas, il faut toutefois soupeser avec soin les risques et les avantages.

6. Les enfants qui se rendent dans une région où l'EJ est endémique, en particulier s'ils y séjournent longtemps, risquent fort d'être infectés par le VEJ et de présenter des complications sérieuses. Bien qu'aucun vaccin contre l'EJ ne soit homologué pour les enfants au Canada, on pourrait envisager une utilisation d'IXIAROMD « non conforme à l'étiquette » ou le recours à un vaccin homologué contre l'EJ dans le pays à risque visité; si l'on recourt à l'une de ces options, on doit toutefois en soupeser avec soin les risques et les avantages. 


\section{Annexe A}

\section{Risque d'encéphalite japonaise par pays ${ }^{\dagger(2)}$}

REMARQUE : Comme la situation à l'égard du risque d'EJ peut changer avec le temps, il est recommandé aux praticiens en médecine des voyages de consulter de l'information à jour sur le risque, par exemple sur le site Web des CDC des États-Unis².

\begin{tabular}{|c|c|c|c|}
\hline Pays & Régions à risque & Saison de transmission & Commentaires \\
\hline Australie & $\begin{array}{l}\text { Îles extérieures du détroit de } \\
\text { Torrès. }\end{array}$ & $\begin{array}{l}\text { De décembre à mai; } \\
\text { tous les cas humains sont } \\
\text { signalés de février à avril. }\end{array}$ & $\begin{array}{l}\text { Un cas humain a été signalé sur le continent, dans } \\
\text { le Nord Queensland. }\end{array}$ \\
\hline Bangladesh & $\begin{array}{l}\text { Peu de données disponibles; } \\
\text { probablement répandue. }\end{array}$ & $\begin{array}{l}\text { Inconnue; la plupart des } \\
\text { cas humains sont signalés } \\
\text { de mai à octobre. }\end{array}$ & $\begin{array}{l}\text { Une éclosion de maladie chez l'humain a été } \\
\text { signalée dans le district de Tangail en } 1977 . \text { La } \\
\text { surveillance sentinelle a permis de répertorier des } \\
\text { cas humains dans les divisions de Chittagong, } \\
\text { Khulna et Rajshahi et le district de Mymensingh. }\end{array}$ \\
\hline Bhoutan & Aucune donnée. & Aucune donnée. & \\
\hline $\begin{array}{l}\text { Birmanie } \\
\text { (Myanmar) }\end{array}$ & $\begin{array}{l}\text { Données limitées; on } \\
\text { présume que la maladie est } \\
\text { endémique dans tout le pays. }\end{array}$ & $\begin{array}{l}\text { Inconnue; la plupart des } \\
\text { cas humains sont signalés } \\
\text { de mai à octobre. }\end{array}$ & $\begin{array}{l}\text { Des éclosions chez l'humain ont été signalées dans } \\
\text { l'État de Shan. Des anticorps anti-VEJ ont été décelés } \\
\text { chez des animaux et l'humain dans d'autres régions. }\end{array}$ \\
\hline Brunei & $\begin{array}{l}\text { Aucune donnée; on présume } \\
\text { que la maladie est endémique } \\
\text { dans tout le pays. }\end{array}$ & $\begin{array}{l}\text { Inconnue; on présume que } \\
\text { la transmission se produit } \\
\text { toute l'année. }\end{array}$ & \\
\hline
\end{tabular}

Cambodge On présume que la maladie est endémique dans tout le pays.
Probablement toute l'année avec des pics signalés de mai à octobre.
La surveillance sentinelle a permis de répertorier des cas humains dans au moins 14 provinces, dont celles de Phnom Penh, Takeo, Kampong, Cham, Battambang, Svay Rieng et Siem Reap.

\begin{tabular}{llll}
\hline Chine & $\begin{array}{l}\text { Cas humains signalés dans } \\
\text { toutes les provinces sauf } \\
\text { celles de Xizang (Tibet), } \\
\text { Xinjiang et Qinghai. }\end{array}$ & $\begin{array}{l}\text { La plupart des cas humains } \\
\text { sont signalés d'avril à } \\
\text { octobre. }\end{array}$ & $\begin{array}{l}\text { Les taux les plus élevés sont déclarés dans les } \\
\text { provinces du sud-ouest et du centre-sud. }\end{array}$ \\
& $\begin{array}{l}\text { Hong Kong et Macao : Non } \\
\text { considérés comme des zones } \\
\text { d'endémie. De rares cas sont } \\
\text { signalés dans les nouveaux } \\
\text { territoires. }\end{array}$ & $\begin{array}{l}\text { Le vacin n'est pas systémement recommandé } \\
\text { aux voyageurs qui se rendent uniquement à } \\
\text { Beijing ou dans d'autres grandes villes. }\end{array}$ \\
\hline $\begin{array}{l}\text { Corée du } \\
\text { Nord }\end{array}$ & Aucune donnée. & Aucune donnée. & \\
\hline
\end{tabular}




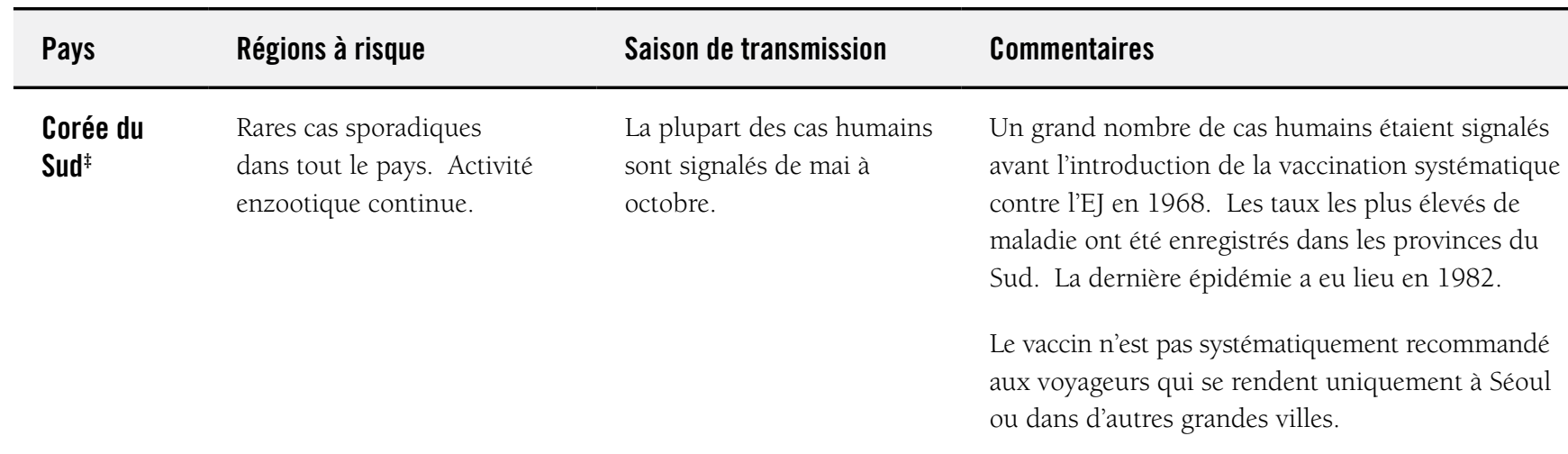

\begin{tabular}{llll}
\hline $\begin{array}{l}\text { Îles du } \\
\text { Pacifique }\end{array}$ & Éclosions de maladie chez & Inconnue; la plupart des \\
Ouest & $\begin{array}{l}\text { l'humain signalées à Guam } \\
\text { en 1947-1948 et à Saipan } \\
\text { en } 1990 .\end{array}$ & $\begin{array}{l}\text { Le cycle enzootique pourrait ne pas être durable; des } \\
\text { d'octobre à mars. }\end{array}$ & \\
& & & \\
\hline
\end{tabular}

\begin{tabular}{|c|c|c|c|}
\hline Inde & $\begin{array}{l}\text { Cas humains signalés dans } \\
\text { tous les États, sauf les } \\
\text { suivants : Dadra, Daman, Diu, } \\
\text { Gujarat, Himachal, Jammu, } \\
\text { Cachemire, Lakshadweep, } \\
\text { Meghalaya, Nagar Haveli, } \\
\text { Punjab, Rajasthan et Sikkim. }\end{array}$ & $\begin{array}{l}\text { La plupart des cas humains } \\
\text { sont signalés de mai à } \\
\text { octobre, particulièrement } \\
\text { dans le nord du pays. La } \\
\text { saison peut être prolongée } \\
\text { ou s'étendre sur toute } \\
\text { l'année dans certaines } \\
\text { régions, en particulier } \\
\text { du sud du pays. }\end{array}$ & $\begin{array}{l}\text { Les taux les plus élevés de maladie chez l'humain } \\
\text { sont signalés dans les États suivants : Andhra } \\
\text { Pradesh, Assam, Bihar, Goa, Haryana, Karnataka, } \\
\text { Kerala, Tamil Nadu, Uttar Pradesh et Bengale- } \\
\text { Occidental. }\end{array}$ \\
\hline Indonésie & $\begin{array}{l}\text { On présume que la maladie } \\
\text { est endémique dans tout le } \\
\text { pays. }\end{array}$ & $\begin{array}{l}\text { Des cas humains sont } \\
\text { signalés toute l'année; le } \\
\text { pic saisonnier varie d'une } \\
\text { île à l'autre. }\end{array}$ & $\begin{array}{l}\text { La surveillance sentinelle a permis de répertorier } \\
\text { des cas humains dans les provinces suivantes: Bali, } \\
\text { Kalimantan, Java, Nusa Tenggara, Papouasie et } \\
\text { Sumatra. }\end{array}$ \\
\hline Japon & $\begin{array}{l}\text { Rares cas sporadiques sur } \\
\text { toutes les îles sauf l'île } \\
\text { d'Hokkaido. Activité } \\
\text { enzootique continue. }\end{array}$ & $\begin{array}{l}\text { La plupart des cas humains } \\
\text { sont signalés de mai à } \\
\text { octobre. }\end{array}$ & $\begin{array}{l}\text { Un grand nombre de cas humains étaient signalés } \\
\text { avant l'introduction de la vaccination systématique } \\
\text { contre l'EJ en 1968. La plupart des petites éclosions } \\
\text { ont été déclarées dans le district de Chugoku } \\
\text { en } 2002 \text {. Des cas sporadiques ont été signalés } \\
\text { parmi le personnel militaire américain sur l'île } \\
\text { d'Okinawa. Une transmission enzootique sans } \\
\text { cas humain a été observée sur l'île d'Hokkaido. } \\
\text { Le vaccin n'est pas systématiquement recommandé } \\
\text { aux voyageurs qui se rendent uniquement à Tokyo } \\
\text { ou dans d'autres grandes villes. }\end{array}$ \\
\hline Laos & $\begin{array}{l}\text { Aucune donnée; on présume } \\
\text { que la maladie est endémique } \\
\text { dans tout le pays. }\end{array}$ & $\begin{array}{l}\text { On présume qu'elle s'étend } \\
\text { de mai à octobre. }\end{array}$ & \\
\hline
\end{tabular}




\begin{tabular}{|c|c|c|c|}
\hline Pays & Régions à risque & Saison de transmission & Commentaires \\
\hline Malaisie & $\begin{array}{l}\text { Endémique dans l'État de } \\
\text { Sarawak; cas et éclosions } \\
\text { sporadiques signalés dans } \\
\text { tous les États de la péninsule, } \\
\text { et probablement dans l'État } \\
\text { de Sabah. }\end{array}$ & Toute l’année. & $\begin{array}{l}\text { La plupart des cas humains sont signalés dans les } \\
\text { États de Penang et Sarawak. } \\
\text { Le vaccin n'est pas systématiquement recommandé } \\
\text { aux voyageurs qui se rendent uniquement à Kuala } \\
\text { Lumpur ou dans d'autres grandes villes. }\end{array}$ \\
\hline Mongolie & $\begin{array}{l}\text { Pas considérée comme un } \\
\text { pays d'endémie. }\end{array}$ & & \\
\hline Népal & $\begin{array}{l}\text { Endémique dans les basses } \\
\text { terres du sud (Terai). Cas } \\
\text { et éclosions sporadiques } \\
\text { signalés dans la vallée de } \\
\text { Kathmandu. }\end{array}$ & $\begin{array}{l}\text { La plupart des cas humains } \\
\text { sont signalés de mai à } \\
\text { novembre. }\end{array}$ & $\begin{array}{l}\text { Les taux les plus élevés de maladie chez l'humain } \\
\text { sont signalés dans les districts de l'Ouest de Teraï, } \\
\text { dont Bankey, Bardia, Dang et Kailali. } \\
\text { Le vaccin n'est pas systématiquement recommandé } \\
\text { aux voyageurs qui se rendent uniquement dans } \\
\text { des régions en haute altitude. }\end{array}$ \\
\hline Pakistan & $\begin{array}{l}\text { Données limitées; cas humains } \\
\text { signalés autour de Karachi. }\end{array}$ & $\begin{array}{l}\text { La plupart des cas humains } \\
\text { sont signalés de mai à } \\
\text { octobre. }\end{array}$ & \\
\hline $\begin{array}{l}\text { Papouasie- } \\
\text { Nouvelle- } \\
\text { Guinée }\end{array}$ & $\begin{array}{l}\text { Données limitées; cas humains } \\
\text { sporadiques signalés dans les } \\
\text { provinces suivantes : Ouest, } \\
\text { Golfe et Hautes-Terres } \\
\text { méridionales. }\end{array}$ & Inconnue. & $\begin{array}{l}\text { Un cas d'EJ a été signalé près de Port Moresby en } \\
\text { 2004. Des cas humains ont été confirmés dans la } \\
\text { province indonésienne de Papouasie. }\end{array}$ \\
\hline Philippines & $\begin{array}{l}\text { Données limitées; on présume } \\
\text { que la maladie est endémique } \\
\text { sur toutes les îles. }\end{array}$ & $\begin{array}{l}\text { Inconnue; probablement } \\
\text { toute l'année. }\end{array}$ & $\begin{array}{l}\text { Des éclosions ont été signalées dans les endroits } \\
\text { suivants : Nueva Ecija, Luzon et Manille. }\end{array}$ \\
\hline Russie & $\begin{array}{l}\text { Rares cas humains signalés } \\
\text { dans les régions maritimes de } \\
\text { l'extrême est situées au sud } \\
\text { de Khabarousk. }\end{array}$ & $\begin{array}{l}\text { La plupart des cas humains } \\
\text { sont signalés de juillet à } \\
\text { septembre. }\end{array}$ & \\
\hline Singapour & $\begin{array}{l}\text { Rares cas humains signalés } \\
\text { de façon sporadique. }\end{array}$ & Toute l'année. & La vaccination systématique n'est pas recommandée. \\
\hline Sri Lanka & $\begin{array}{l}\text { Endémique dans tous } \\
\text { le pays, sauf en régions } \\
\text { montagneuses. }\end{array}$ & $\begin{array}{l}\text { Toute l'année, avec des } \\
\text { pics variables selon les } \\
\text { pluies de la mousson. }\end{array}$ & $\begin{array}{l}\text { Les taux les plus élevés de maladie chez l'humain sont } \\
\text { signalés dans les districts suivants : Anuradhapura, } \\
\text { Gampaha, Kurunegala, Polonnaruwa et Puttalam. }\end{array}$ \\
\hline
\end{tabular}




\begin{tabular}{|c|c|c|c|}
\hline Pays & Régions à risque & Saison de transmission & Commentaires \\
\hline Taïwan & $\begin{array}{l}\text { Rares cas humains } \\
\text { sporadiques sur toute l'île. }\end{array}$ & $\begin{array}{l}\text { La plupart des cas humains } \\
\text { sont signalés de mai à } \\
\text { octobre. }\end{array}$ & $\begin{array}{l}\text { Un grand nombre de cas humains étaient signalés } \\
\text { avant l'introduction de la vaccination systématique } \\
\text { contre l'EJ en } 1968 \text {. } \\
\text { Le vaccin n'est pas systématiquement recommandé } \\
\text { aux voyageurs qui se rendent uniquement à Taipei } \\
\text { ou dans d'autres grandes villes. }\end{array}$ \\
\hline Thaïlande & $\begin{array}{l}\text { Endémique dans tout le pays; } \\
\text { épidémies saisonnières dans } \\
\text { les provinces du nord. }\end{array}$ & $\begin{array}{l}\text { Toute l'année, avec des } \\
\text { pics saisonniers de mai } \\
\text { à octobre, en particulier } \\
\text { dans le nord. }\end{array}$ & $\begin{array}{l}\text { Les taux les plus élevés de maladie chez l’humain } \\
\text { sont signalés dans la vallée de Chiang Mai. Des } \\
\text { cas humains sporadiques ont été déclarés dans } \\
\text { les banlieues de Bangkok. }\end{array}$ \\
\hline Timor-Leste & $\begin{array}{l}\text { Données limitées; rapports } \\
\text { non vérifiables de cas } \\
\text { humains sporadiques. }\end{array}$ & Aucune donnée. & \\
\hline Vietnam & $\begin{array}{l}\text { Endémique dans tous le } \\
\text { pays; épidémies saisonnières } \\
\text { dans les provinces du nord. }\end{array}$ & $\begin{array}{l}\text { Toute l'année, avec des } \\
\text { plics saisonniers de mai } \\
\text { à octobre, en particulier } \\
\text { dans le nord. }\end{array}$ & $\begin{array}{l}\text { Les taux les plus élevés de maladie sont signalés } \\
\text { dans les provinces du nord autour de Hanoï et } \\
\text { dans les provinces du nord-ouest ayant une } \\
\text { frontière commune avec la Chine. }\end{array}$ \\
\hline
\end{tabular}




\section{Annexe B}

\section{Distribution géographique de l'encéphalite japonaise ${ }^{(2)}$}

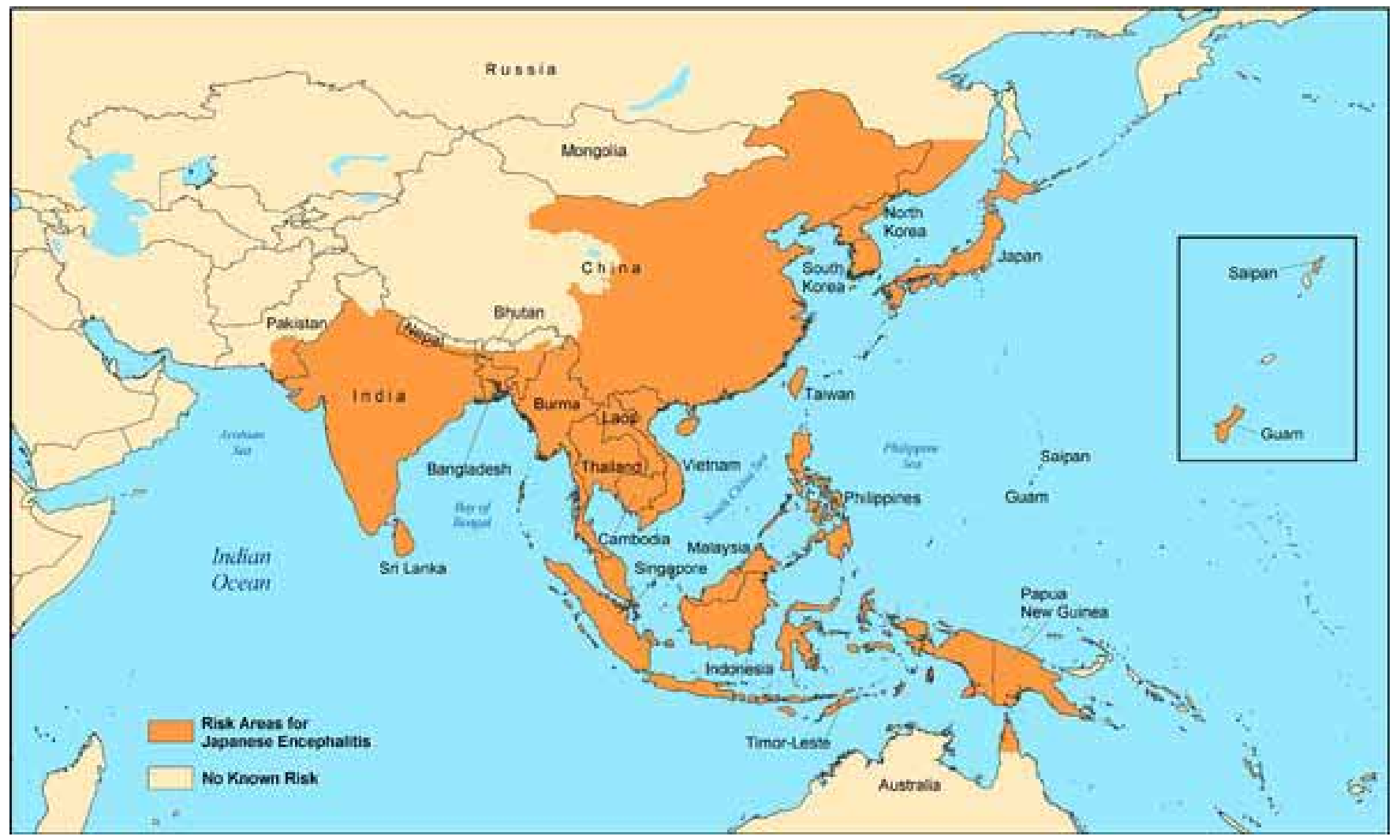




\section{Annexe C}

\section{Facteurs à considérer lorsqu'on évalue le risque d'exposition au virus de I'encéphalite japonaise (VEJ) chez un voyageur ${ }^{(3)}$}

\section{Destination}

LEJ sévit dans presque tous les pays d'Asie et certaines parties du Pacifique Ouest.

C'est dans les régions rurales agricoles, souvent les régions dans lesquelles on produit du riz ou dans lesquelles on procède à l'irrigation par inondation, que le risque d'exposition au VEJ est le plus élevé.

De vastes éclosions localisées d'EJ peuvent survenir, ce qui indique une transmission très active du VEJ dans la région touchée.

\section{Durée du voyage}

La plupart des cas d'EJ associée aux voyages qui sont signalés sont des expatriés ou encore des voyageurs qui effectuent un long séjour ( $\geq 1$ mois) dans une région à risque.

Bien qu'on ne puisse définir une durée précise de séjour qui entraîne un risque pour le voyageur, un long séjour augmente la probabilité d'exposition à un moustique infecté par le VEJ.

\section{Saison}

Dans la plupart des régions tempérées d'Asie, la transmission du VEJ est saisonnière et la maladie chez l'humain atteint habituellement son point culminant l'été et l'automne.

Dans les régions subtropicales ou tropicales, le profil de transmission du VEJ varie, et les cas humains peuvent être sporadiques ou être répertoriés à longueur d'année.

\section{Activités}

Les moustiques qui transmettent le VEJ se nourrissent de sang humain, le plus souvent à l'extérieur. Les moments de la journée où ils se nourrissent sont surtout après le coucher du soleil, puis après minuit.

Les activités extérieures de longue durée (camping, randonnée, randonnée en montagne, bicyclette, pêche, chasse, agriculture, etc.), en particulier le soir ou la nuit, augmentent le risque d'exposition à un moustique infecté par le VEJ.

Les logements sans climatisation, moustiquaires ni moustiquaires de lit augmentent le risque d'exposition aux moustiques qui transmettent le VEJ et d'autres maladies vectorielles (p. ex. dengue et paludisme). 


\section{Utilisation de mesures de protection individuelle}

Lutilisation de MPI (moustiquaire de lit, insectifuge, vêtements traités, etc.) devrait offrir une protection substantielle contre les piqûres de moustiques qui transmettent le VEJ. La mesure dans laquelle les MPI sont respectées peut influer beaucoup sur le risque d'exposition au VEJ. 


\section{Annexe D}

\section{Fermeté et qualité des preuves ${ }^{(30)}$}

\section{Catégories relatives à la fermeté de chaque recommandation}

\begin{tabular}{cl}
\hline CATÉGORIE & DÉFINITION \\
\hline A & Preuves suffisantes pour recommander l'utilisation. \\
\hline B & Preuves acceptables pour recommander l'utilisation. \\
\hline C & Preuves insuffisantes pour recommander ou déconseiller l'utilisation. \\
\hline D & Preuves acceptables pour déconseiller l'utilisation. \\
\hline E & Preuves suffisantes pour déconseiller l'utilisation. \\
\hline
\end{tabular}

\section{Catégories relatives à la qualité des preuves sur lesquelles reposent les recommandations}

\begin{tabular}{cl}
\hline CLASSE & DÉFINITION \\
\hline I & Données obtenues dans le cadre d'au moins un essai comparatif convenablement randomisé. \\
\hline II & $\begin{array}{l}\text { Données obtenues dans le cadre d'au moins un essai clinique bien conçu, sans randomisation, d'études de cohortes } \\
\text { ou d'études analytiques cas-témoins, réalisées de préférence dans plus d'un centre, à partir de plusieurs séries } \\
\text { chronologiques, ou résultats spectaculaires d'expériences non comparatives. }\end{array}$ \\
\hline III & $\begin{array}{l}\text { Opinions exprimées par des sommités dans le domaine et reposant sur l'expérience clinique, des études descriptives } \\
\text { ou des rapports de comités d'experts. }\end{array}$ \\
\hline
\end{tabular}




\section{Références}

1. World Health Organization. WHO Position Paper: Japanese encephalitis vaccines. Wkly Epidemiol Record 2006;81:331-40.

2. Centers for Disease Control and Prevention. CDC Health Information for International Travel 2010. Atlanta: U.S. Department of Health and Human Services, Public Health Service, 2009.

3. Advisory Committee on Immunization Practices (ACIP). Japanese encephalitis vaccines. MMWR 2010;59(RR-1).

4. Vaughn DW, Hoke CH. The epidemiology of Japanese encephalitis: Prospects for prevention. Epidemiol Rev 1992;14:197-221.

5. Hoke $\mathrm{CH}$, Nisalak A, Sangawhipa $\mathrm{N}$ et al. Protection against Japanese encephalitis by inactivated vaccines. N Engl J Med 1988;319:608-14.

6. Luo D, Zhang K, Song J, Yao R et al. The protective effect of bed nets impregnated with pyrethroid insecticides and vaccination against Japanese encephalitis. Trans R Soc Trop Med Hyg 1994;88:632-34.

7. Solomon T, Nguyen MD, Kneen R, Gainsboroug M et al. Neurological aspects of tropical disease: Japanese encephalitis. J Neurol Neurosurg Psychiatry 2000;68:405-15.

8. Hills SL, Griggs AC and Fischer M. Japanese encephalitis in travelers from non-endemic countries, 1973-2008. Am J Trop Med Hyg 2010;82(5):930-36.

9. Freedman DO, Weld LH, Kozarsky PE, Fisk T et al. Spectrum of disease and relation to place of exposure among ill returned travelers. N Engl J Med 2006;354:119-30.

10. Boggild AK, Castelli F, Gautret P, Torresi J et al. Vaccine preventable diseases in returned travelers: results from the GeoSentinel Surveillance Network. Vaccine 2010;28(46):7389-95.

11. Artsob H, Spence L. Imported arbovirus infections in Canada, 1974-89. Can J Infect Dis 1991;2:95-100.

12. Centers for Disease Control and Prevention. Japanese encephalitis in a US traveler returning from Thailand, 2004. MMWR 2005;54(5):123-25.

13. Frances SP, Eamsila C, Pilakasiri $C$ et al. Effectiveness of repellent formulations containing DEET against mosquitoes in northeastern Thailand. J Am Mosq Control Assoc 1996;12:331-33.

14. Thavara U, Tawatsin A, Chompoosri J et al. Laboratory and field evaluations of the insect repellent 3535 (ethyl butylacetylaminopriopionate) and DEET against mosquito vectors in Thailand. J Am Mosq Control Assoc 2001;17:190-95.

15. Jinjiang X, Meiluan Z, Xinfu L et al. Evaluation of permethrin-impregnated mosquito-nets against mosquitoes in China. Med Vet Entomol 1988;2:247-51.

16. Harbach RE, Tang DB, Wirtz RA et al. Relative repellency of two formulations of N,N-diethyl-3-methylbenzamide (DEET) and permethrin-treated clothing against Culex sitiens and Aedes vigilax in Thailand. J Am Mosq Control Assoc 1990;6:641-44.

17. Committee to Advise on Tropical Medicine and Travel (CATMAT). Statement on personal protective measures to prevent arthropod bites. CCDR 2005;31(ACS-4). 
18. Health Canada. Notice of decision for IXIARO ${ }^{\circledR}$ [Internet]. 2009 Dec 2 [cited 2010 Jul 12]. Available from: http://www. hc-sc.gc.ca/dhp-mps/prodpharma/sbd-smd/phase1-decision/drug-med/nd_ad_2009_ixiaro_120330-eng.php.

19. Intercell AG. Product monograph: IXIARO ${ }^{\circledast}:$ Japanese encephalitis vaccine (inactivated, adsorbed; suspension for injection [Internet]. 2009 Sep 15 [cited 2010 Jul 13]. Available from: http://www.novartis.ca/downloads/en/products/ ixiaro_monograph_en.pdf.

20. Kaltenbock A, Dubischar-Kastner K, Schuller E, Datla M et al. Immunogenicity and safety of IXIARO (IC51) in a Phase II study in healthy Indian children between 1 and 3 years of age. Vaccine 2010;28(3):834-39.

21. US Food and Drug Administration. March 30, 2009 Approval letter [for IXIARO ${ }^{\circledR}$ ] [Internet]. 2009 Mar 30 [updated 2009 Apr 30; cited 2010 Jul 13]. Available from: http://www.fda.gov/BiologicsBloodVaccines/Vaccines/ ApprovedProducts/ucm 142577.htm.

22. European Medicines Agency. European public assessment report: IXIARO [Internet]. 2010 [updated 2010 Apr 20; cited 2010 Jul 30]. Available from: http://www.ema.europa.eu/ema/index.jsp?curl=pages/medicines/human/ medicines/000963/human_med_000862.jsp\&murl=menus/medicines/medicines.jsp\&mid=WC0b0 1ac058001d125.

23. Tauber E, Kollaritsch H, Korinek M, Rendi-Wagner P et al. Safety and immunogenicity of a Vero-cell-derived inactivated Japanese encephalitis vaccine: a non-inferiority phase III, randomised controlled trial. Lancet 2007;370:1847-53.

24. Tauber E, Kollaritsch H, von Sonnenburg F, Lademann M et al. Randomized, double-blind, placebo-controlled Phase 3 trial of the safety and tolerability of IC51, and inactivated Japanese encephalitis vaccine. JID 2008;198:493-99.

25. Schuller E, Klade CS, Wolfl G, Kaltenbock A et al. Comparison of a single, high-dose vaccination regimen to the standard regimen for the investigational Japanese encephalitis vaccine, IC51: a randomized, observer-blind, controlled Phase 3 study. Vaccine 2009;27(15):2188-93.

26. Dubischar-Kaster K, Eder S, Buerger V, Gartner-Woelfl G et al. Long-term immunity and immune response to a booster dose following vaccination with the inactivated Japanese encephalitis vaccine IXIARO, IC51. Vaccine 2010;28(32):5197-202.

27. Schuller E, Jilma B, Voicu V, Golor G et al. Long-term immunogenicity of the new Vero cell-derived inactivated Japanese encephalitis virus vaccine IC51: six and 12 mois results of a multicenter follow-up phase 3 study. Vaccine 2008;26(34):4382-86.

28. Kaltenbock A, Dubischar-Kastner K, Eder G, Jilg W et al. Safety and immunogenicity of concomitant vaccination with the cell-culture based Japanese encephalitis vaccine IC51 and the hepatitis A vaccine HAVRIX 1440 in healthy subjects: a single-blind, randomized, controlled phase 3 study. Vaccine 2009;27(33):4483-89.

29. Public Health Agency of Canada. Canadian immunization guide, $7^{\text {th }}$ ed., 2006.

30. Committee to Advise on Tropical Medicine and Travel. Evidence-based medicine. RMTC 1994;20:145-47. 